\title{
Synthesis and characterization of hierarchical mesoporous NaY zeolite templated with a novel multi-headed quaternary ammonium salt surfactant
}

\author{
Jialin $\mathrm{Yu}$ *, Dong $\mathrm{Xu}$ \\ Beijing Key Laboratory of Functional Material in Power Generation System, Guodian New Energy \\ Technology Research Institute, Beijing 102209, China \\ ${ }^{*}$ Corresponding author: yujialin@cgdc.com.cn
}

Keywords: Hierarchically porous zeolite $\mathrm{Y}$, new surfactant template, mesopore, micropore, synthesis.

\begin{abstract}
An approach for the synthesis of hierarchical mesoporous zeolite $\mathrm{NaY}$, templated with Bis [2-hydroxy-3-(dodecyldimethylammonio) propyl]-diethylammonium Trichloride, is reported. The results show that the meseoporous zeolite $\mathrm{NaY}$ possesses both micro and mesopore system, and the mesopore size distributed at around $2.8 \mathrm{~nm}$ and $5.8 \mathrm{~nm}$. Moreover, the detailed structure difference from the synthesis conditions such as crystallization temperature and template amount is detected, and the optimal conditions are proposed.
\end{abstract}

\section{Introduction}

Microporous molecular sieves, such as Y, Beta and ZSM-5, are important materials due to their wide applications as adsorbents, ion exchangers and catalyst components [1, 2]. However, the pore size of these zeolites results in a large diffusion barrier for large dimensional molecules. The ordered mesoporous materials, such as MCM-41 [3], express the low acidity and poor thermal stability. Therefore, the ideal hierarchically mesoporous zeolite should possess the intrinsic properties of conventional zeolites and an auxiliary mesopore network, which facilitates the diffusion of the large reactant molecules.

In recent years, much work have been published on the topics discussing the strategies to create mesoporosity in the microporous materials [4]. Groen et al. [5] applied the desilication with a base leaching method to create mesopores in ZSM-5. Ogura et al. [6] applied desilicated mesoporous ZSM-5 in cumene cracking reaction. Wang et al. [7] published a facile synthesis method of a hierarchical mesoporous ZSM-5 zeolite from a starch-derived bread-template. Xiao and his coworkers [8] synthesized a hierarchically mesoporous Beta zeolite using poly diallyl dimethyllammonium chloride (PDADMAC) as the template, and the material has higher activity and sulfur-tolerance in aromatic compound hydrogenation than other traditional zeolite as the support. Schüth et al. [9] disclosed a synthetic pathway for fabricating of a monolithic silicalite-1 with hierarchical pore systems using organic aerogel template from polymerization of resorcinol and formaldehyde.

In this work, Gemini cationic surfactant, Bis [2-hydroxy- 3- (dodecyldimethylammonio) propyl]-diethylammonium Trichloride, is used as the mesoporous template for the first time. Zeolite $\mathrm{Y}$ with hierarchical pore structure is synthesized at low hydrothermal temperature.

\section{Experimental}

\subsection{Y zeolite precursor synthesis}

A Y precursor suspension is prepared as in ref. [10]. $6.7 \mathrm{~g}$ sodium hydroxide (Tianjin Chemical Co.), 1.67 g sodium aluminate (Tianjin Chemical Co.), and $40 \mathrm{ml} \mathrm{H}_{2} \mathrm{O}$ are mixed. Then $21.6 \mathrm{~g} 25$ wt\% silica sol (Tianjin Chemical Co.) is added. After stirring at $95{ }^{\circ} \mathrm{C}$ for $12 \mathrm{~h}$ and aged at room temperature for several hours, zeolite $\mathrm{Y}$ seed suspension is obtained. 


\subsection{Mesoporous Zeolite Y synthesis}

The 3-tailed quarternary ammonium salt $\left(\mathrm{C}_{38} \mathrm{H}_{84} \mathrm{~N}_{3} \mathrm{O}_{2} \mathrm{Cl}_{3}\right)$ (Dingzhou Radi-Glory Bio-Chem Co.,Ltd ) is used as the template, and the molecular structure is shown as followed.<smiles>CCCCC(O)C(O)C[N+](C)(C)CC[N+](C)(C)CCCl</smiles>

The precursor suspension is mixed with different amount template $16 \mathrm{~g}$ at room temperature. After stirring for $24 \mathrm{~h}$, the slurry is loaded into an autoclave and kept at $60^{\circ} \mathrm{C}$ for 5 days. The as-prepared crystalline solid is filtrated, washed with distilled water, and dried in air at $110{ }^{\circ} \mathrm{C}$. Finally, the product is calcined at $550{ }^{\circ} \mathrm{C}$ in air for $8 \mathrm{~h}$. The samples are denoted as Y-T-x-M-y, where $\mathrm{x}$ means synthesis temperature and y means surfactant amount. As a comparison, we also get a commercial sample NaY from Nankai University Catalyst Co.

\subsection{Characterization}

The X-ray diffraction (XRD) patterns are measured with a Brucker-AXS D8 Focus X'pert diffractometer using $\mathrm{Cu} \mathrm{K} \alpha$ radiation $(\lambda=0.15406 \mathrm{~nm})$ at $40 \mathrm{kV}$ and $40 \mathrm{~mA}$. The nitrogen adsorption-desorption isotherms (NADIs) are measured at $-196{ }^{\circ} \mathrm{C}$ with a Quantachrome Autosorb-1. The mesopore size distribution is calculated from the adsorption branch of the isotherm using the Barrett-Joyner-Halenda (BJH) method. The t-plot curves are obtained to calculate the micropore volume and the micropore area through the adsorption branch according to ref. [11]. The micropore size distribution is calculated from density function theory (DFT) method. High-resolution transmission electron microscopy (HRTEM) is obtained with a PHILIPS TECNAI G ${ }^{2}$ F20 model with an acceleration voltage of $200 \mathrm{kV}$. Scanning election microscope (SEM) images are recorded on a Hitachi S-4800 instrument.

\section{Results and discussion}

Fig. 1 shows the XRD pattern of the sample Y-T-60-M-16 in the high $2 \theta$ region 5-50o. As shown, the diffraction peaks corresponds to those of zeolite $Y$. No reflection is found in the small $2 \theta$ region, indicating there is no structural order in the mesopore scale.

The nitrogen adsorption isotherms in Fig. 2 (a) exhibit type IV isotherms with H4 rectangular type hysteresis loops at $0.5<\left(\mathrm{P} / \mathrm{P}_{0}\right)<0.9$, besides large amount of nitrogen adsorbed at $\mathrm{P} / \mathrm{P}_{0}<0.02$ [12]. Fig. 2 (b) shows mesopore-size distribution from BJH model and micropore-size distribution from DFT model. BJH pore size distribution is centered at $2.8 \mathrm{~nm}$ and $5.6 \mathrm{~nm}$ narrowly, confirming the presence of mesopore. It is noted that DFT pore size distribution is narrowly centered at $0.54 \mathrm{~nm}$, which is assigned to the micropores of FAU crystals.

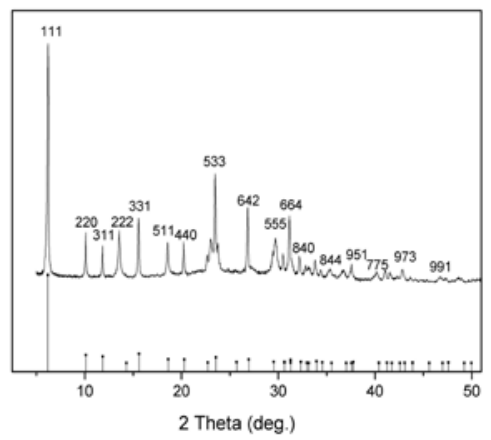

Fig. 1 The XRD pattern of the sample Y-T-60-M-16

Fig. 4 gives the SEM images of the samples. Different from the conventional zeolite $Y$ with regular shape and smooth surface in Fig. 4c and d, the mesoporous zeolite Y shows irregular shape with large size particles. Higher magnification image (Fig. 4b) reveals that the large particle surface is loose and rough, constructed of primary zeolite crystals with various sizes. The observed 
mesoporosity are formed from the intergrowth of zeolite nano-seed particles. In Fig. 5, the crystalline lattice fringe of zeolite Y corresponding to the (111) crystal surface, can be easily observed, and the interplanar spacing is $1.40 \mathrm{~nm}$, again revealing the high crystallinity of this sample.

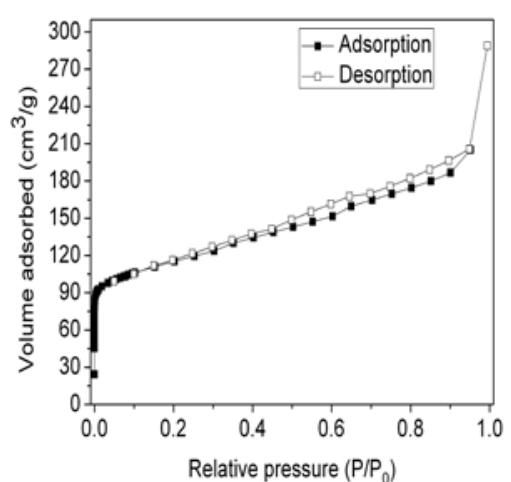

(a)

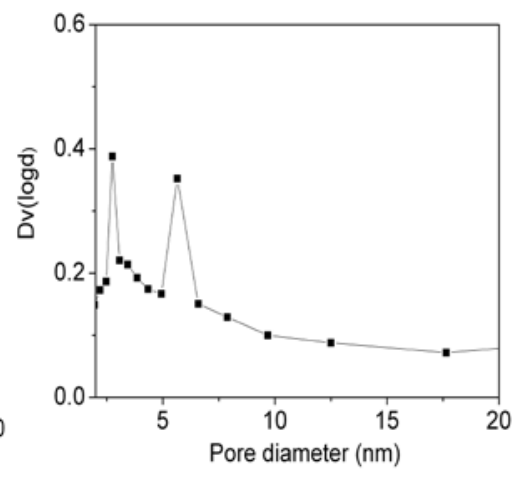

(b)

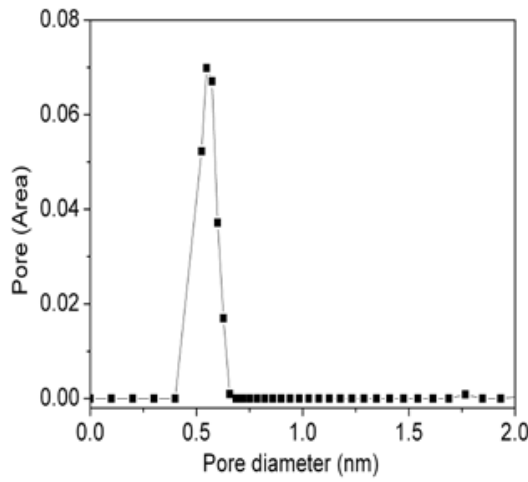

(c)

Fig. 2 Pore analysis of sample Y-T-60-M-16: (a) nitrogen adsorption-desorption isotherm;

(b) mesopore size distribution; (c) Micropore size distribution

The obtained mesoporous zeolite Y has the characteristic diffraction peaks associated with MFI structure, indicating the successful crystallization. The nitrogen adsorption-desorption isothermal reveals the porosity in hierarchical mesoporous zeolite Y. The mesoporous zeoliteY exhibited a major uptake occurred at low relative pressures less than 0.02 and a broad hysteresis loop basically at relative pressure of $0.5-0.9$, indicating the co-presence of micropores and mesopores. The H4 type hysteresis loop can also be deduced that the mesopore in the new material is narrow channel type [11]. The pore size distribution plots confirm the mesopore size and micropore size. Nitrogen adsorption analysis reveals that there are a large number of mesopores in the sample. The BET surface area and mesopore volume is $446 \mathrm{~m}^{2} / \mathrm{g}$ and $0.43 \mathrm{ml} / \mathrm{g}$.
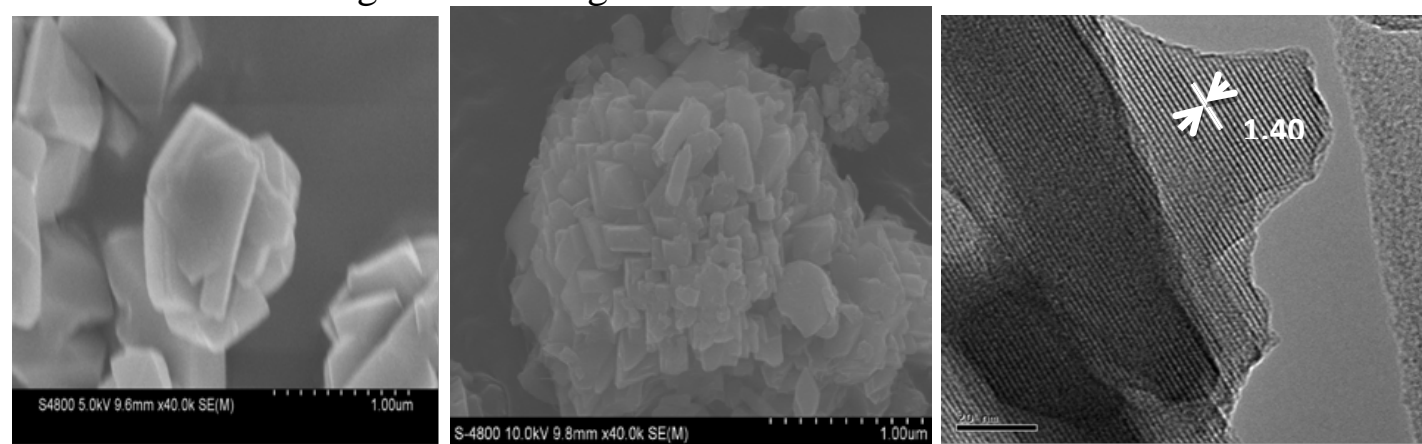

Figure 3 The morphology of sample Y-T-60-M-16: (a) Zeolite Y; (b) Y-T-60-M-16; (c) HTEM

SEM and HTEM images display the morphology intuitively. The particle is about $1 \mu \mathrm{m}$ with rough surface composed of nanosized crystals, and the mesoporosity is formed from the aggregation of nanosized particles, which also agrees with the nitrogen adsorption results. These results indicate that the mesoporous zeolite $\mathrm{Y}$ is a polycrystalline zeolite with mesoposity and that the surface tends to recrystallize into larger crystals through organic template attachment.

Assembly of zeolite nanocrystals into hierarchically materials has gathered a lot of research interests. The formation of mesoporous zeolite can be depicted as shown in schematic 1. Primary zeolite nanocrystals are formed in the initial reaction period and they assemble into polycrystalline particles through organic template attachment in the hydrothermal reaction condition. The imperfect intergrowth of the zeolite nanocrystals causes the observed mesoporosity. The nanoparticles in the center assemble with each other, whereas the nanocrystals on the surface of the aggregate grow with amorphous material through organic template attachment during crystallization. The recrystallization reaction will continue from the outer surface to the inner part of the aggregate. Mesoporous aggregates of zeolite nanocrystals will form if the amount of amorphous material in the synthesis solution is mall. 


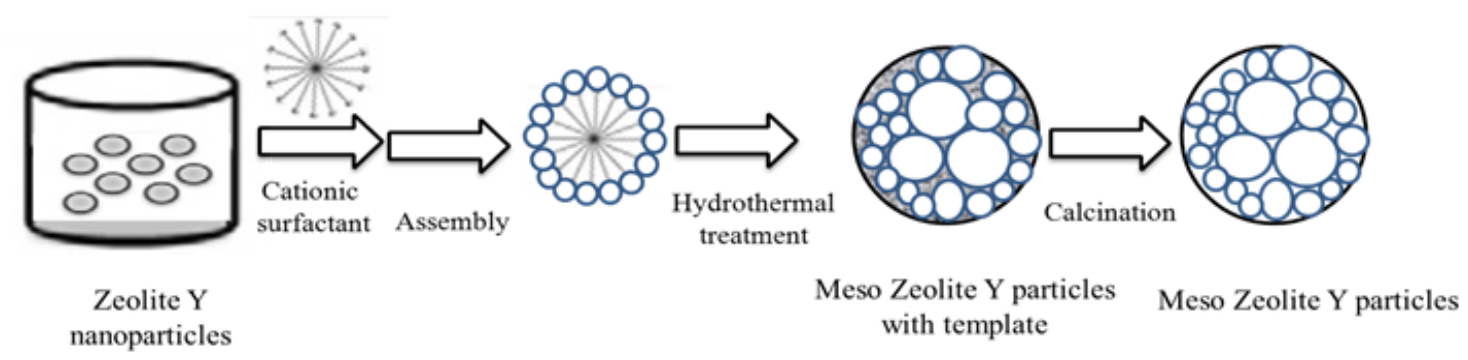

Scheme 1. Schematic representation of the synthesized meso zeolite Y

\section{Conclusion}

Mesoporous zeolite $\mathrm{NaY}$ is synthesized by a novel method with Bis [2-hydroxy-3(dodecyldimethylammonio) propyl]-diethylammonium Trichloride as template firstly. The results showed that the new material contained both micro- and mesopore system, and the mesopore size distributed at around $2.8 \mathrm{~nm}$ and $5.8 \mathrm{~nm}$. In our view, such the synthesis of zeolites comprising desired mesoporous structures will become the focus of much attention and the target in zeolite research field on the quest for novel mesoporous materials.

\section{References}

[1] A. Corma. Inorganic solid acids and their use in acid-catalyzed hydrocarbon reactions. Chem. Rev., 1995, 3, 559-614.

[2] J. Čejka and Wichterlová B. Acid-catalyzed synthesis of mono- and dialkyl benzenes over zeolites: active sites, zeolite topology, and reaction mechanism. Catal. Rev., 2002, 44, 375-421.

[3] J.S. Beck, J.C. Vartuli, W.J. Roth, M.E. Leonowicz, C.T. Kresge, K.D. Schmitt, C.T.W. Chu, D.H. Olson, E.W. Sheppard, S.B. McCullen, J.B. Higgins, J.L. Schlenker. A new family of mesopours molecular-sieves prepared with liquid-crystal templates. J. Am. Chem. Soc. 1992, 114, 10834-10843. [4] J. Kim , M. Choi, R. Ryoo. Effect of mesoporosity against the deactivation of MFI zeolite catalyst during the methanol-to-hydrocarbon conversion process. J. Catal., 2010, 269, 1, 219-228.

[5] D.P. Serrano, J.M. Escola, P. Pizarro. A sol-gel approach for the room temperature synthesis of Al-containing micelle-templated silica, Microporous Mesoporous Mater. 2012, 34, 1, 43-54.

[6] J.C. Groen, L.A.A. Peffer, J.A. Moulijn, J. Perez-Ramirez. On the introduction of intracrystalline mesoporosity in zeolites upon desilication in alkaline medium. Microporous Mesoporus Mater. 2004, 69, 29-34.

[7] L.F. Wang, C.Y. Yin, Z.C. Shan, S. Liu, Y.C. Du, F.S. Xiao. Bread-template synthesis of hierarchical mesoporous ZSM-5 zeolite with hydrothermally stable mesoporosity. Colloids Surf. A, 2009, 340, 1-3, 126-130.

[8] F.S. Xiao, L.F. Wang, C.Y. Yin, K.F. Lin, Y. Di, J.X. Li, R.R. Xu, D.S. Su, R. Schlgl, T. Yokoi, T. Tatsumi. Catalytic properties of hierarchical mesoporous zeolites templated with a mixture of small organic ammonium salts and mesoscale cationic polymers. Angew. Chem. Int. Ed. 2006, 45(19), 3090-3093.

[9] W.C. Li, A.H. Lu, R. Palkovits, W. Schmidt, B. Spliethoff, F.Schüth. Hierarchically structured monolithic silicalite-1 consisting of crystallized nanoparticles and its performance in the Beckmann rearrangement of cyclohexanone oxime. J. Am. Chem. Soc. 2005, 127(36), 12595-12600.

[10] Y. Liu, W. Zhang, T.J. Pinnavaia. Steam-stable aluminosilicate mesostructures assembled from zeolite type Y seeds. J. Am. Chem. Soc. 2000, 122, 8791-8792.

[11] B.C. Lippens, J.H. Boer. Studies on pore systems in catalysts: V. The t method. J. Catal., 1965, 4, 3, 319-323.

[12] S.J. Greg, K.S.W. Sing. Adsorption, surface area and porosity. Academic Press: London, 1982. 